\title{
Differences of the crystallization and diffusion trends by Fo-Ni variations
}

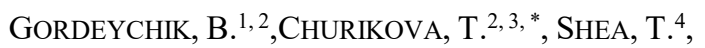 \\ KRONZ, A. ${ }^{2}$, SIMAKIN, A. ${ }^{1,5}$ AND WÖRNER, G. ${ }^{2}$ \\ ${ }^{1}$ Institute of Experimental Mineralogy, RAS, Chernogolovka, \\ 142432, Russia \\ ${ }^{2}$ Geowissenschaftliches Zentrum Göttingen, Abteilung \\ Geochemie, Universität Göttingen, Göttingen, 37077, \\ Germany \\ ${ }^{3}$ Institute of Volcanology and Seismology, FEB RAS, \\ Petropavlovsk-Kamchatsky, 683006, Russia \\ (*correspondence: tchurikova@mail.ru) \\ ${ }^{4}$ Department of Earth Sciences, University of Hawaii, \\ Honolulu, HI 96822, USA \\ ${ }^{5}$ Institute of Physics of the Earth, RAS, Moscow, 123242, \\ Russia
}

$\mathrm{Ni}$ is a compatible element in olivine, so crystallization typically results in a concave-up trend on a Fo-Ni diagram. We consider Ni-enriched olivine phenocrysts compositions that fall above a crystallization trend. To explain the origin Ni-enriched olivine crystals, we develop a set of models to describe the diffusion in olivine phenocrysts formed in primitive parent (high-Mg, high-Ni) melt and trapped in an evolved (low-Mg, low-Ni) melt. These models describe the loss of $\mathrm{Fo}$ and $\mathrm{Ni}$ in olivine cores during protracted diffusion. If the diffusivity of $\mathrm{Ni}$ is lower than that for Fo then olivine phenocrysts affected by diffusion form a concave-down trend that contrasts with the concave-up crystallization trend. Models for different simple crystals geometries show that concave-down curvature of the diffusion trend does not depend on the size of the crystals and only weakly depends on their shape and their anisotropy. Numerical 3-D diffusion models using realistic olivine morphologies with anisotropy corroborate this conclusion. The curvature of the diffusion trend is determined by the ratio of $\mathrm{Fo}$ and $\mathrm{Ni}$ diffusion coefficients only. The initial and final points of the diffusion trend are determined by the compositional contrast between mafic and more evolved melts. We present several examples of measurements on olivine from arc basalts from Kamchatka, and several published olivine datasets from mafic magmas from non-subduction settings (lamproites and kimberlites) that are consistent with diffusion-controlled Fo-Ni behaviour. In all examples the ratio of Fo and Ni diffusion coefficients is indicated to be $<1$. These examples show that crystallization and diffusion can be distinguished by concave-up and concave-down trends in Fo-Ni diagrams. This research was supported by DFG grant No. Wo 362/51-1, and RFBR grants No. 16-55-12040 and 17-55-50005. 